\title{
Tumor Lysis Syndrome Risk in Outpatient Versus Inpatient Administration of Venetoclax and Hypomethlators for Acute Myeloid Leukemia
}

\section{Ari Pelcovits}

Brown University Warren Alpert Medical School

Jozal Moore

University of Rochester School of Medicine and Dentistry

Brianna Bakow

Brown University Warren Alpert Medical School

\section{Rabin Niroula}

Brown University Warren Alpert Medical School

\section{Pamela Egan}

Brown University Warren Alpert Medical School

John Reagan ( $\square$ john_reagan@brown.edu )

Brown University Warren Alpert Medical School https://orcid.org/0000-0003-4877-6863

\section{Research Article}

Keywords: Tumor Lysis, Acute Leukemia, Venetoclax, Hypomethylator

Posted Date: February 22nd, 2021

DOI: https://doi.org/10.21203/rs.3.rs-160517/v1

License: (9) (i) This work is licensed under a Creative Commons Attribution 4.0 International License.

Read Full License

Version of Record: A version of this preprint was published at Supportive Care in Cancer on March 4th, 2021. See the published version at https://doi.org/10.1007/s00520-021-06119-7. 


\section{Abstract}

\section{Introduction}

Venetoclax along with hypomethylating agents (HMAs) is the new standard therapy for older patients with acute myeloid leukemia (AML) not fit for intensive frontline induction chemotherapy. Venetoclax is associated with fatal episodes of tumor lysis syndrome (TLS) in chronic lymphocytic leukemia (CLL), and recommendations are for its initiation for CLL and AML in the inpatient setting with close monitoring. Herein, we evaluated the safety of outpatient venetoclax ramp up when given in addition to HMAs for the treatment of AML.

\section{Methods}

We conducted a retrospective review of patients diagnosed with AML at our institution from 12/1/2016 until $7 / 1 / 2020$. We identified patients who received HMAs and venetoclax for AML, either as frontline or relapsed/refractory therapy. Records were reviewed for evidence of laboratory or clinical tumor lysis episodes in all patients.

\section{$\underline{\text { Results }}$}

Between 12/1/2016 and 7/1/2020 43 patients at our institution received venetoclax/HMA for the treatment of AML. Thirty-nine patients $(91 \%)$ had venetoclax initiation and ramp up in the outpatient setting.

One episode of laboratory TLS (2.5\%) was identified. This patient required admission to the hospital for rasburicase and IV fluids with resolution of the laboratory effects without resultant clinical TLS. There were no episodes of clinical TLS in either group. 30-day mortality from venetoclax initiation was $0 \%$ in both groups.

\section{Conclusion}

Our experience with HMAs and venetoclax showed that outpatient ramp up of venetoclax is safe with a very low risk of laboratory TLS (2.5\%) and no evidence of clinical TLS within our cohort.

\section{Introduction}

Acute myeloid leukemia (AML) is an aggressive malignancy of white blood cells driven by clonal proliferation of myeloid precursor cells. Five-year overall response to therapy among young patients $(<55)$ can be as high as $64 \%$ in favorable risk groups with standard of care chemotherapy [1]. With a median age at diagnosis of 68 however, the treatment of older adults with AML is an important and ongoing area of research [2]. Older adults tend to have significantly worse outcomes, with five-year overall survival rate in those 65 and older of $8.2 \%$, and only $3.2 \%$ specifically in those 75 and older [2]. 
For older patients not fit enough to tolerate high intensity induction chemotherapy. the standard of care until recently was either low dose cytarabine or the use of hypomethylating agents such as azacitidine or decitabine [3]. Hypomethylating agents have been shown in clinical trials to improve median overall survival compared to conventional care regimens, however median overall survival still frequently remains less than 1 year, despite a reduction in chemotherapy related mortality $[4,5]$. With the hope of improving remission rates and overall survival while still maintaining the benefit of less toxic chemotherapy several targeted agents have been investigated. With promising phase 1 and now phase 3 results, the addition of venetoclax to hypomethylating agents is quickly becoming the new standard of care for elderly patients not fit enough for induction chemotherapy [6-8].

Venetoclax is an oral, highly selective inhibitor of the anti-apoptotic protein BCL-2. BCL2 is involved in mitochondrial cell regulation of apoptosis and may play a role in cell response to chemotherapy in patients with AML [9]. Venetoclax was therefore investigated for use in combination with hypomethylating agents (decitabine or azacitadine) in a Phase 1 trial and ultimately in a Phase 3 randomized control trial comparing azacitadine plus venetoclax vs. azacitdine alone in older adults with newly diagnosed acute myeloid leukemia $[6,7]$. The addition of venetoclax improved median overall survival from 9.6 to 14.1 months [7].

While this regimen allows patients to avoid the morbidity and mortality of standard induction chemotherapy, the addition of venetoclax comes with concerns of unique increased toxicity. Initially used in chronic lymphocytic leukemia (CLL), venetoclax is associated with fatal episodes of tumor lysis syndrome in early CLL trials $[10,11]$. Package insert and expert opinion recommendations therefore in the treatment of $A M L$ are for its initiation in the inpatient setting, with a dose escalation of $100 \mathrm{mg}$ day 1 , $200 \mathrm{mg}$ day 2 , and $400 \mathrm{mg}$ day 3 with IV hydration and close monitoring of tumor lysis markers $[12,13]$. In the initial phase $1 \mathrm{~b}$ trial that resulted in its approval for use in patients with $A M L$, as well as in the Phase 3 trial, all patients were admitted to the hospital for initial venetoclax ramp up for close tumor lysis monitoring $[6,7]$.

At our institution the practice has been to initiate HMA/VEN as an outpatient provided the white blood cell count (WBC) is $<25 \times 10^{9} / \mathrm{L}$. Herein, we evaluated the safety of outpatient venetoclax ramp up when given in addition to hypomethylating agents for the treatment of acute myeloid leukemia.

\section{Methods}

\section{Data}

We conducted a retrospective review of patients diagnosed with AML at our institution from 12/1/2016 until $7 / 1 / 2020$ and who received either decitabine or azacitidine in combination with venetoclax.

\section{Patient Population}


We included patients aged 18 and older who received the above therapies at any point in their illness, frontline for newly diagnosed disease or as salvage therapy for relapse or refractory leukemia. We stratified patients based on whether venetoclax was initiated in the inpatient or outpatient setting.

\section{Outcomes}

We examined baseline AML characteristics including initial white blood cell count, blast count, cytogenetics and molecular profiles as well as baseline TLS markers (potassium, calcium, phosphate, uric acid, and creatinine) prior to the initiation of venetoclax. Using the Cairo-Bishop Criteria as modified by Howard we examined the number of episodes of laboratory or clinical tumor lysis in all patients [14].

TLS as defined by the Cairo-Bishop Criteria as modified by Howard is stratified into laboratory and clinical tumor lysis. Laboratory tumor lysis is defined as having at least two of four alterations in serum electrolyte or uric acid values during the same 24-hour period in the 3 days prior to or up to 7 days following treatment of a malignancy. These are: 1) Uric Acid level of $>8.0 \mathrm{mg} / \mathrm{dl} 2$ ) Phosphorous $>4.5$ $\mathrm{mg} / \mathrm{dl} \mathrm{3)} \mathrm{Potassium}>6.0 \mathrm{mmol} /$ liter and 4) Corrected calcium of $<9.0 \mathrm{mg} / \mathrm{dl}$ or ionized calcium $<4.5$ $\mathrm{mg} / \mathrm{dl}$. Acute kidney injury is not an aspect of laboratory TLS.

Clinical TLS is defined as the presence of laboratory tumor lysis in addition to one of 3 clinical scenarios. These are: 1) Cardiac dysrhythmia or sudden death felt likely or definitely to be caused by hyperkalemia or hypocalcemia 2) Seizure, neuromuscular irritability, hypotension, or heart failure felt likely or definitely to have been caused by hypocalcemia and 3) Acute kidney injury as defined by an increase in serum creatinine of $0.3 \mathrm{mg} / \mathrm{dl}$ or if no baseline value is present than a value of $>1.5$ times the upper limit of normal, or oliguria defined as $<0.5 \mathrm{ml} / \mathrm{kg} / \mathrm{hr}$ of urine output over 6 hours.

Baseline TLS labs in our study were collected in all patients. As the majority of patients had initiation of venetoclax in the outpatient setting many did not have values within 3 days of starting treatment. In these patients the values most recently obtained prior to initiating venetoclax were used as baseline values. Similarly, not all patients had lab values obtained within 1 week of starting venetoclax. For the purpose of this study it was assumed these patients did not have laboratory or clinical tumor lysis. Thirty-day mortality from initiation of venetoclax was measured to account for possible unidentified episodes of clinical tumor lysis.

As noted above laboratory tumor lysis requires the presence of at least two lab abnormalities. We additionally made note of patients with only one lab abnormality to broaden our analysis to include patients who may have been at risk of developing but did not meet criteria of laboratory or clinical tumor lysis.

\section{Statistical Methods}

Fisher's exact test and Wilcoxon rank-sum tests were performed to examine differences in categorical and continuous variables. 


\section{IRB Approval}

This retrospective chart review study involving human participants was in accordance with the ethical standards of the institutional and national research committee and with the 1964 Helsinki Declaration and its later amendments or comparable ethical standards. The Human Investigation Committee (IRB) of Rhode Island Hospital approved this study.

\section{Results}

Between 12/1/2016 and 7/1/2020 43 patients at our institution received venetoclax in addition to a hypomethylating agent for the treatment of AML (Table 1). Twenty-four patients received azacitadine, 11 decitabine, and 8 received both hypomethylators at some point during therapy. Twenty-two patients received hypomethylators and venetoclax as frontline treatment while the other 21 received it as salvage therapy. Thirty-nine patients (91\%) had venetoclax initiation and ramp up in the outpatient setting. There were 28 patients who received venetoclax within 28 days of starting the hypomethylating agent, 25 of whom received it as an outpatient and three as an inpatient.

AML characteristics were as follows: 21 pts were diagnosed with de novo AML, 16 with secondary AML, and 7 with therapy related AML. Twenty pts had a normal karyotype and 14 pts had a complex karyotype. Patients had a wide array of mutations as would be expected in an older population with the only significant difference in patients with DNMT3a more likely to be admitted to the hospital for venetoclax initiation ( $p$-value of 0.02 , Table 2).

Pretreatment labs were notable for median normal values of potassium, phosphate, uric acid, calcium and creatinine (Table 1). The median pretreatment creatinine levels between the two groups were not significantly different. Median pre-treatment white blood cell count (WBC) was noted to be significantly higher in the inpatient cohort (37.9 vs $5.5 \times 10^{9} / \mathrm{L}$, p-value of 0.01$)$.

Amongst all patients there was only one identified episode of laboratory TLS (2.5\%) with an elevated phosphate and uric acid. This occurred in the outpatient group in a patient whose pretreatment WBC count was greater than $25 \times 10^{9} / \mathrm{L}$. This patient required admission to the hospital for rasburicase and IV fluids with resolution of the laboratory effects without resultant clinical TLS. There were no episodes of clinical TLS in either group. Thirty-day mortality from venetoclax initiation was $0 \%$ in both groups.

While the Cairo-Bishop Criteria require the presence of two lab abnormalities to diagnose TLS we did a further analysis to evaluate for the presence of even a single lab abnormality associated with TLS. We identified three additional patients $(7.5 \%)$ in the outpatient cohort who had the presence of only one lab abnormality associated with TLS within the seven days after initiating treatment. None of these patients required any further TLS directed treatment or hospitalization.

\section{Discussion}


In this single institution retrospective analysis, we found that venetoclax was able to be safely ramped up in the outpatient setting, with low risk of tumor lysis and no episodes of fatal toxicity, in patients with AML. This is the first study to our knowledge that has evaluated the safety of outpatient venetoclax ramp up in patients with AML.

The concern regarding tumor lysis with venetoclax stems from its use in chronic lymphocytic leukemia (CLL). In two of the initial phase 1 trials examining the role of venetoclax in CLL, one with venetoclax monotherapy and the other in combination with rituximab, both had a single episode of fatal tumor lysis $[10,11]$. This led to modifications in dose administration including ramp up dose escalation and tumor lysis monitoring, after which no further fatal episodes of tumor lysis were seen in either trial.

For this reason, randomized control trials and expert opinion regarding the use of venetoclax in AML have required or recommended inpatient monitoring for dose escalation given the potential risk of tumor lysis syndrome [15]. In these studies, the rates of tumor lysis were low; in the initial phase 1 trial no patients were found to have clinical or laboratory tumor lysis [6]. Patients though were required to have an absolute white blood cell count of $<25 \times 10^{9} / \mathrm{L}$ and all patients were hospitalized. In the phase 3 analysis only $1 \%$ of patients experienced tumor lysis, which resolved with intervention, but similarly all patients were admitted for monitoring during dose escalation of venetoclax [7]. Despite this low incidence of TLS expert opinion remains that dose escalation for venetoclax ramp up in AML should be done in the inpatient setting given the concerns regarding TLS in the CLL literature [13].

Limitations of this study include its retrospective design, single institution cohort and incomplete tumor lysis labs for all patients before and immediately following venetoclax ramp up. Incomplete labs were due to the retrospective nature of the design, as patients were not required in our institution to have specific tumor lysis labs drawn prior to or immediately following initiation of venetoclax. While the true incidence of laboratory tumor lysis may be higher amongst our patient population due to this missing data, the rate of clinical tumor lysis is likely similar as clinically significant events, such as arrythmias, seizures, or renal failure, would likely have precipitated need for further care and work up. Furthermore, the 30-day mortality for patients following initiation of venetoclax was 0 , indicating that even if there were missed episodes of tumor lysis none of these were fatal.

Our study demonstrates that venetoclax can be safely ramped up in the outpatient setting for the treatment of AML. The risk of tumor lysis remains low, specifically clinical tumor lysis, and especially amongst patients with white blood cell counts less than $25 \times 10^{9} / \mathrm{L}$.

\section{Conclusion}

In this single institution retrospective analysis outpatient ramp up of venetoclax in addition to hypomethylating agents for the treatment of AML is safe with a very low risk of laboratory TLS $(2.5 \%)$ and no evidence of clinical TLS. We therefore conclude that venetoclax in combination with 
hypomethylators for AML treatment can be safely initiated as an outpatient once the white blood cell count has been reduced below $25 \times 10^{9} / \mathrm{L}$.

\section{Declarations}

Funding: The authors did not receive support from any organization for the submitted work

Conflicts of interest/Competing interests: The authors have no relevant financial or non-financial interests to disclose.

Code availability: Not applicable

Authors' contributions: All authors made substantial contributions to the conception or design of the work, acquisition, analysis, or interpretation of data, drafting the work or revising it critically for important intellectual content, approved the version to be published, and agreed to be accountable for all aspects of the work in ensuring that questions related to the accuracy or integrity of any part of the work are appropriately investigated and resolved.

Ethics approval: This retrospective chart review study involving human participants was in accordance with the ethical standards of the institutional and national research committee and with the $1964 \mathrm{Helsinki}$ Declaration and its later amendments or comparable ethical standards. The Human Investigation Committee (IRB) of Rhode Island Hospital approved this study.

Consent to participate: Not applicable

Consent for publication: Not applicable

Availability of data and material: Not applicable

Code availability: Not applicable

\section{References}

1. Grimwade WH, Oliver D, Wheatley F, Harrison K, Harrison C, Rees G, Hann J, Stevens I, Burnett R, Goldstone A A, "The Importance of Diagnostic Cytogenetics on Outcome in AML: Analysis of 1,612 Patients Entered Into the MRC AML 10 Trial," Blood, vol. 92, no. 7, pp. 2323-2333, 1998

2. "National Cancer Institute Surveillance, Epidemiology, and End Results Program Cancer Stat Facts: Leukemia-Acute Myeloid Leukemia (AML) Vol 2020."

3. MA Sekeres et al., "American Society of Hematology 2020 Guidelines for Treating Newly Diagnosed Acute Myeloid Leukemia in Older Adults" Blood Adv, vol. 4, no. 15, pp. 3528-3549, Aug 112020

4. P Fenaux et al (Feb 12010 ) "Azacitidine prolongs overall survival compared with conventional care regimens in elderly patients with low bone marrow blast count acute myeloid leukemia". J Clin Oncol 
28(4):562-569

5. H Dombret et al., "International phase 3 study of azacitidine vs conventional care regimens in older patients with newly diagnosed AML with > 30\% blasts," Blood, vol. 126, no. 3, pp. 291-9, Jul 162015

6. DiNardo PK, Pullarkat CD, Jonas V, Arellano BA, Becker M, Frankfurt PS, Konopleva O, Wei M, Kantarjian AH, Xu HM, Hong T, Chyla WJ, Potluri B, Pollyea J, Letai DA A, "Venetoclax combined with decitabine or azacitidine in treatment-naive, elderly patients with acute myeloid leukemia," Blood, vol. 133, no. 1, pp. 7-17, 2019

7. CD DiNardo et al., "Azacitidine and Venetoclax in Previously Untreated Acute Myeloid Leukemia" $N$ Engl J Med, vol. 383, no. 7, pp. 617-629, Aug 132020

8. "National Comprehensive Cancer Network. Acute Myeloid Leukemia (V3.2020)."

9. TT Vo et al., "Relative mitochondrial priming of myeloblasts and normal HSCs determines chemotherapeutic success in AML," Cell, vol. 151, no. 2, pp. 344 - 55, Oct 122012

10. JF Seymour et al (2017) "Venetoclax plus rituximab in relapsed or refractory chronic lymphocytic leukaemia: a phase 1b study". The Lancet Oncology 18(2):230-240

11. AW Roberts et al., "Targeting BCL2 with Venetoclax in Relapsed Chronic Lymphocytic Leukemia," $N$ Engl J Med, vol. 374, no. 4, pp. 311 - 22, Jan 282016

12. "U.S. Food and Drug Administration. Highlights of Prescribing Information: Venclexta; 2018."

13. Jonas BA, Pollyea DA, "How we use venetoclax with hypomethylating agents for the treatment of newly diagnosed patients with acute myeloid leukemia," Leukemia, vol. 33, no. 12, pp. 2795-2804, Dec 2019

14. Howard JD, Pui S (2011) CH "The Tumor Lysis Syndrome". N Engl J Med 364:1844-1854

15. LE Roeker et al., "Tumor Lysis, Adverse Events, and Dose Adjustments in 297 Venetoclax-Treated CLL Patients in Routine Clinical Practice" Clin Cancer Res, vol. 25, no. 14, pp. 4264-4270, Jul 152019

\section{Tables}

Table 1: Patient clinical and laboratory characteristics divided by receipt of venetoclax inpatient versus outpatient. 


\begin{tabular}{|llll|}
\hline Patient Characteristics & $\begin{array}{l}\text { Outpatient VEN } \\
\text { Ramp Up } \\
(\mathbf{N}=39)\end{array}$ & $\begin{array}{l}\text { Inpatient VEN } \\
\text { Ramp Up } \\
(\mathbf{N}=4)\end{array}$ & $\begin{array}{l}\text { p- } \\
\text { value }\end{array}$ \\
\hline Age, Mean & $70(52-88)$ & $78(56-80)$ & 0.3 \\
\hline Sex, Female & $16(41 \%)$ & $2(50 \%)$ & 1.00 \\
\hline Frontline & $20(51 \%)$ & $2(50 \%)$ & 1.00 \\
\hline ECOG 0-1 & $5(13 \%)$ & $0(0 \%)$ & 1.00 \\
\hline ECOG 2 & $34(87 \%)$ & $4(100 \%)$ & 1.00 \\
\hline Charlson Comorbidity Index & $5(3-8)$ & $6(3-6)$ & 0.41 \\
\hline Blast Count (Pre-Treatment Bone Marrow & $20 \%(0.9-82)$ & $51.5 \%(29-74)$ & 0.23 \\
\hline Biopsy Percent) & & & \\
\hline Pretreatment: & & $3.55(3.4-4)$ & 0.05 \\
Potassium (mEq/L) & $\mathbf{4 ( 3 . 2 - 5 . 1 )}$ & $2.45(1.9-2.6)$ & 0.03 \\
Phosphate (mg/dL) & $3.6(3.5-3.7)$ & $3.95(3.6-5.3)$ & 0.009 \\
Uric Acid (mg/dL) & $5.65(3.2-7.9)$ & $8.75(8.6-9.3)$ & 0.27 \\
Calcium (mg/dL) & $9.2(8.2-12.2)$ & $1.035(0.9-1.65)$ & 0.5 \\
\hline Creatinine (mg/dL) & $0.98(0.46-1.82)$ & & \\
\hline
\end{tabular}

Table 2: Patient AML specific characteristics divided by receipt of venetoclax inpatient versus outpatient. 


\begin{tabular}{|llll|}
\hline AML Characteristics & Outpatient VEN Ramp Up & Inpatient VEN Ramp Up & p-value \\
\hline AML Subtype & & & \\
De Novo & $18(46 \%)$ & $2(50 \%)$ & 1.00 \\
Secondary & $15(39 \%)$ & $1(25 \%)$ & 1.00 \\
Therapy Related & $6(15 \%)$ & $1(25 \%)$ & 0.52 \\
\hline Cytogenetics & & & \\
Normal Karyotype & $19(50 \%)$ & $1(25 \%)$ & 0.61 \\
Complex Karyotype & $13(34 \%)$ & $1(25 \%)$ & 1.00 \\
Molecular & & & 1.00 \\
TP53 & $12(36 \%)$ & $1(25 \%)$ & 0.1 \\
FLT3 & $4(11 \%)$ & $2(50 \%)$ & 0.02 \\
DNMT3A & $4(12 \%)$ & $3(75 \%)$ & 0.52 \\
TET2 & $5(15 \%)$ & $1(25 \%)$ & 1.00 \\
NPM1 & $7(21 \%)$ & $1(25 \%)$ & 0.56 \\
IDH & $6(17 \%)$ & $1(25 \%)$ & 1.00 \\
ASXL1 & $8(24 \%)$ & $1(25 \%)$ & \\
\hline
\end{tabular}

*Cytogenetic data was not available for one patient who received outpatient venetoclax ramp up.

\#Molecular data was available for 33 patients who received outpatient venetoclax ramp up and all 4 patients who received inpatient venetoclax ramp up. 